\title{
Endometrial Metastasis from Breast Cancer during Adjuvant Endocrine Therapy
}

\author{
Fumikata Hara ${ }^{a}$ Sachiko Kiyoto ${ }^{a}$ Daisuke Takabatake \\ Seiki Takashima ${ }^{a}$ Kenjiro Aogia Shozo Ohsumi ${ }^{a}$ \\ Norihiro Teramoto ${ }^{\text {b }}$ Rieko Nishimurab \\ Shigemitsu Takashima ${ }^{a}$
}

Departments of a Breast Oncology, and ${ }^{b}$ Pathology, National Hospital Organization

Shikoku Cancer Center, Matsuyama, Japan

\section{Key Words}

Breast cancer - Uterine metastases - Tamoxifen therapy

\begin{abstract}
It is well-known that tamoxifen increases the risk of endometrial cancer. Although metastasis to the uterus from breast cancer is uncommon, there have been some case reports on uterine metastasis. If an endometrial abnormality is detected, the differential diagnosis of whether the uterine tumor is metastatic or primary is very important to determine the course of treatment. We herein report a case in which we detected a uterine tumor during follow-up after treatment with tamoxifen, and demonstrate that GCDFP-15 is useful in diagnosing metastatic uterine tumors arising from breast cancer.
\end{abstract}

\section{Introduction}

Tamoxifen has played a critical role in the treatment of patients with hormone receptor-positive breast cancer [1]. However, it is well-known that tamoxifen increases the risk of endometrial cancer. This side effect occurs as a result of the agonistic effect of tamoxifen on the uterine endometrium. Therefore, gynecologic follow-up is necessary during tamoxifen treatment [2]. Although breast cancer metastasis to the uterus is uncommon, there have been a few case reports on uterine metastasis $[3,4]$. If an endometrial abnormality is detected, differential diagnosis of whether the uterine tumor is metastatic or primary is very important to determine the type of treatment that should be administered. GCDFP-15 is a specific marker for breast cancer, which is very useful in defining whether the primary site was the breast [5]. We herein report a case where we detected a uterine tumor during follow-up after treatment with tamoxifen, and we further 


\begin{tabular}{|c|c|c|c|}
\hline $\begin{array}{l}\text { Cose Reports in } \\
\text { Ona.ayly }\end{array}$ & $\begin{array}{l}\text { Case Rep Oncol 2010;3:137-141 } \\
\text { D0I: } 10.1159 / 000313921\end{array}$ & Published online: April 29, 2010 & $\begin{array}{l}\text { @ } 2010 \text { S. Karger AG, Basel } \\
\text { ISSN 1662-6575 } \\
\text { www.karger.com/cro }\end{array}$ \\
\hline
\end{tabular}

confirm the usefulness of GCDFP-15 as a diagnostic marker for metastatic uterine tumors arising from breast cancer.

\section{Case Report}

A 44-year-old female presented with a mass, $5.5 \times 4.5 \mathrm{~cm}$ in diameter, in her breast, and she was diagnosed with cT3aN1M0 stage IIIa invasive lobular breast carcinoma. Immunohistochemical staining for estrogen receptor was positive, but those for progesterone receptor and human epidermal growth factor receptor-2 were negative. The patients received neoadjuvant chemotherapy with 9 cycles of weekly docetaxel. She underwent a mastectomy and axillary lymph node dissection. Residual tumors remained in both the breast and lymph nodes. She received adjuvant chemotherapy with 4 cycles of adriamycin and cyclophosphamide, and tamoxifen was administered for two years, and then the patient was subsequently switched to anastrozole.

Two months after treatment with anastrozole, the patient complained of abnormal uterine bleeding. Computed-tomography, magnetic resonance imaging (ig. 1) and transvaginal ultrasound revealed that her uterus had thickened endometrium, similar to the increased endometrial proliferation associated with tamoxifen administration. Endometrial curettage was performed. The pathological findings revealed signet ring cell adenocarcinoma, which is not typical of primary uterine tumors (fig. $2 \mathrm{a}$ ). Immunohistochemical staining of the sample was positive for estrogen receptor, GCDFP-15 and CK7, and was negative for CK20 (fig. 2d, b and c, respectively). These findings were consistent with breast cancer that had metastasized to the uterus. Systemic examinations, including upper endoscopy, were performed, and there was no evidence suggesting other primary or metastatic diseases. Thereafter, the patient received systemic chemotherapy. However, she died as a result of pleural carcinomatosis and multiple bone metastasis 11 months after the diagnosis of recurrence.

\section{Discussion}

It is known that breast cancer can metastasize to many organ sites. The most frequently reported metastatic sites are bone, lung and liver, whereas the uterus is an uncommon organ for metastasis from breast cancer. Most of the uterine metastases are found on autopsy [6]. However, among the extragenital cancers, breast cancer is the most common cancer that metastasizes to the uterus, and invasive lobular carcinoma is particularly more likely to metastasize to the uterus [7].

Tamoxifen has played an essential role in the treatment of hormone receptor-positive breast cancer. Based on the data generated during the EBCTCG's meta-analysis, five years of treatment with tamoxifen can reduce the risk of both recurrence and mortality of breast cancer by approximately $30 \%$ [1]. This is caused by the antagonistic effect of tamoxifen on breast cancer cells. However, tamoxifen exerts a partial agonistic effect on the endometrium of the uterus. Therefore, treatment with tamoxifen increases the incidence of endometrial hyperplasias, polyps, and neoplasms. The relative risk of developing endometrial cancer following treatment with tamoxifen is approximately 2-4 times higher than for patients who have not received the drug. These uterine tumors are frequently detected because patients experience unusual uterine bleeding [2]. However, the most common manifestation of uterine metastasis has also been reported to be uterine bleeding [8]. Therefore, it is very difficult to diagnose whether a uterine tumor is a primary tumor arising as a result of tamoxifen treatment, or whether it is a metastatic tumor. In the present case, tamoxifen was used as an adjuvant hormone therapy. Precise diagnosis affects critical decision-making with regard to the treatment of the uterine cancer (i.e. a primary uterine tumor should be resected, while a metastatic uterine tumor should be treated with systemic therapy as a first choice). 


\begin{tabular}{c|l|l|l}
$\begin{array}{c}\text { Cose Reports in } \\
\text { Ondology }\end{array}$ & $\begin{array}{l}\text { Case Rep Oncol 2010;3:137-141 } \\
\text { D01: 10.1159/000313921 }\end{array}$ & Published online: April 29, 2010 & $\begin{array}{l}\text { O 2010 S. Karger AG, Basel } \\
\text { ISSN 1662-6575 } \\
\text { www.karger.com/cro }\end{array}$ \\
\hline
\end{tabular}

In the present case, histology revealed a signet ring morphology. A signet ring morphology is seen in gastrointestinal malignancies, but it can also be seen in breast cancer, in which it is regarded as a subtype of invasive lobular carcinoma with a poor prognosis [9]. According to recent reports, CK20 and CK7 are useful in diagnosing the origin of tumors with signet ring morphology. While gastric cancers are typically CK20 positive and CK7 negative, breast cancers are generally CK20 negative and CK7 positive [10]. GCDFP is another marker known for having high sensitivity and specificity when used for the differential diagnosis of breast cancer [5]. In the present case, immunohistochemical staining of the uterine tumor revealed positivity for GCDFP and CK7, but negative staining for CK20. Therefore, the tumor was defined as being a metastatic tumor from invasive lobular carcinoma of the breast.

Although there have so far only been a few reports on the prognosis of patients with metastatic uterine tumors from breast cancer, the patients in these reports (including the present report) all had a poor prognosis. However, given the limited number of reported cases, further studies are needed, along with a larger number of these cases, to further our understanding of the prognosis of these cancers and to determine the best course of treatment [11].

In conclusion, it should be kept in mind that breast cancer patients who have received tamoxifen may develop not only primary endometrial cancers, but may also sometimes demonstrate uterine metastases.

Fig. 1. Abdominal magnetic resonance imaging revealed diffuse thickening of the uterus.

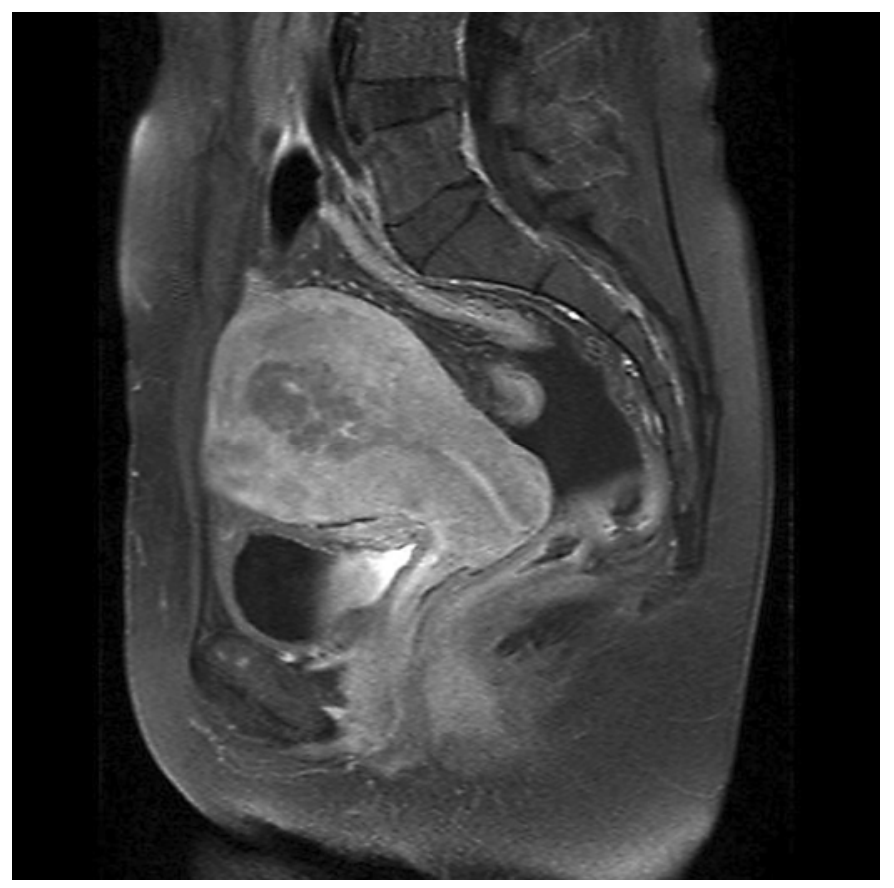




\begin{tabular}{c|l|l|l}
$\begin{array}{c}\text { Cose Reports in } \\
\text { Ondaloyly }\end{array}$ & $\begin{array}{l}\text { Case Rep Oncol 2010;3:137-141 } \\
\text { D0I: 10.1159/000313921 }\end{array}$ & Published online: April 29, 2010 & $\begin{array}{l}\text { I 2010 S. Karger AG, Basel } \\
\text { ISSN 1662-6575 } \\
\text { www.karger.com/cro }\end{array}$ \\
\hline
\end{tabular}

Fig. 2. Hematoxylin and eosin staining of endometrial specimen revealed a poorly differentiated adenocarcinoma with signet ring cell morphology (a). Immunohistochemistry showed strongly positive staining for CK7 (b), negative staining for CK20 (c) and positive staining for GCDFP-15 (d).
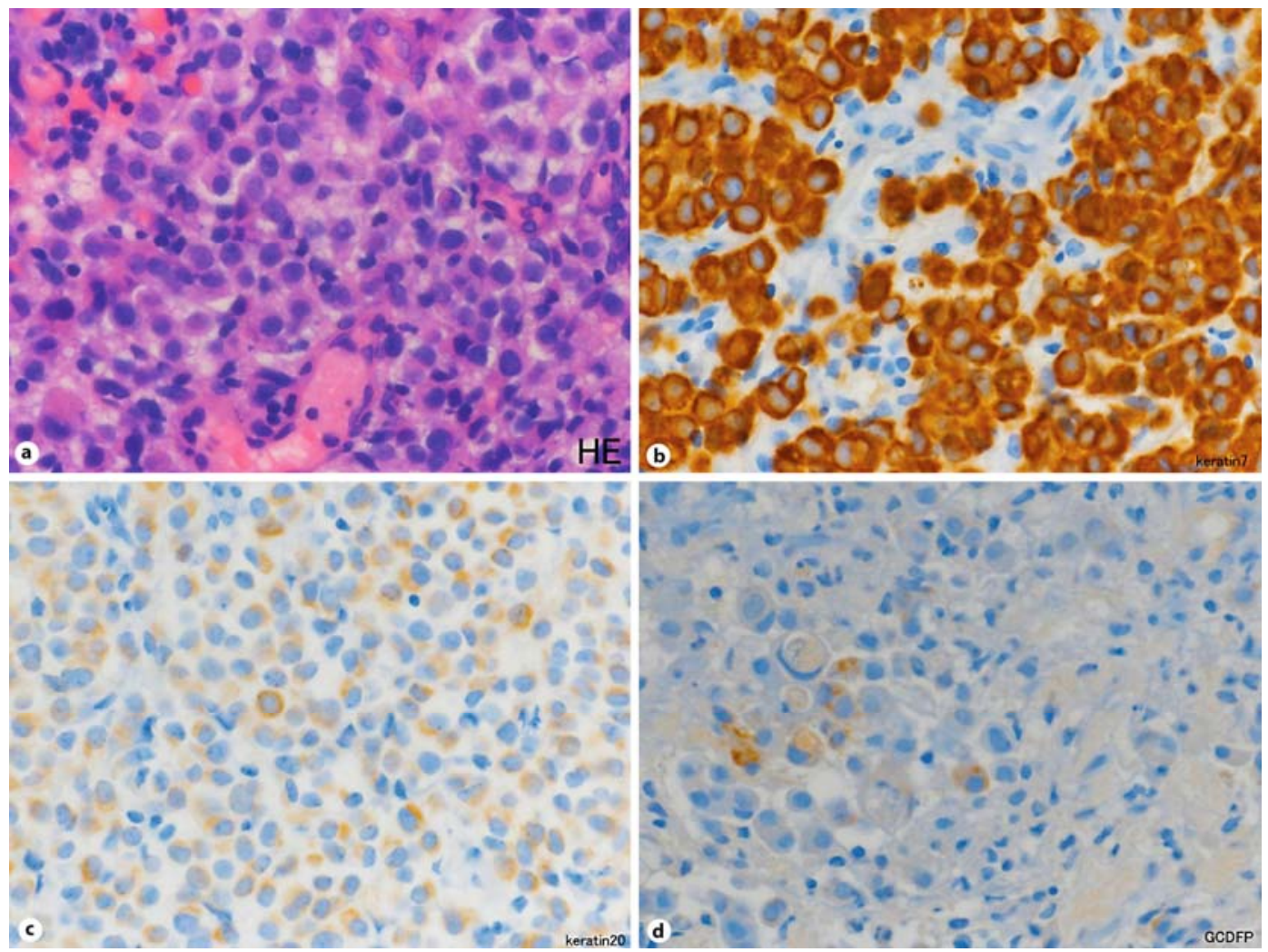


\section{References}

1 Early Breast Cancer Trialists' Collaborative Group (EBCTCG): Effects of chemotherapy and hormonal therapy for early breast cancer on recurrence and 15-year survival: an overview of the randomised trials. Lancet 2005;365:16871717.

-2 Ismail SM: Gynaecological effects of tamoxifen. J Clin Pathol 1999;52:83-88.

-3 Scopa CD, Aletra C, Lifschitz-Mercer B, Czernobilsky B: Metastases of breast carcinoma to the uterus. Report of two cases, one harboring a primary endometrioid carcinoma, with review of the literature. Gynecol Oncol 2005;96:543-547.

4 Manci N, Marchetti C, Esposito F, Graziano M, Tomao F, Pastore M, Bellati F, Panici PB: Late breast cancer recurrence to the uterine cervix with a review of the literature. Int J Gynecol Pathol 2008;27:113-117.

5 Wick MR, Lillemoe TJ, Copland GT, Swanson PE, Manivel JC, Kiang DT: Gross cystic disease fluid protein-15 as a marker for breast cancer: immunohistochemical analysis of 690 human neoplasms and comparison with alpha-lactalbumin. Hum Pathol 1989;20:281-287.

-6 Di Bonito L, Patriarca S, Alberico S: Breast carcinoma metastasizing to the uterus. Eur J Gynaecol Oncol 1985;6:211-217.

7 Lamovec J, Bracko M: Metastatic pattern of infiltrating lobular carcinoma of the breast: an autopsy study. J Surg Oncol 1991;48:8-33.

8 Piura B, Yanai-Inbar I, Rabinovich A, Zalmanov S, Goldstein J: Abnormal uterine bleeding as a presenting sign of metastases to the uterine corpus, cervix and vagina in a breast cancer patient on tamoxifen therapy. Eur J Obstet Gynecol Reprod Biol 1999;83:57-61.

-9 Raju U, Ma CK, Shaw A: Signet ring variant of lobular carcinoma of the breast: a clinicopathologic and immunohistochemical study. Mod Pathol 1993;6:516-520.

10 Tot T: Cytokeratins 20 and 7 as biomarkers: usefulness in discriminating primary from metastatic adenocarcinoma. Eur J Cancer 2002;38:758-763.

-11 Mazur MT, Hsueh S, Gersell DJ: Metastases to the female genital tract. Analysis of 325 cases. Cancer 1984;53:1978-1984. 